\title{
BRAIN-ACTUATED ASSISTIVE MOBILITY FOR DISABLED END-USER
}

\author{
Leeb R, Carlson T, Tonin L and Millán JdR \\ Chair in Non-Invasive Brain-Machine Interface, École Polytechnique Fédérale de Lausanne, Switzerland \\ robert.leeb@epfl.ch
}

\begin{abstract}
Brain-Computer Interfaces (BCIs) have been successfully used to control assistive mobility devices (like a telepresence robot or an electric wheelchair) using only motor imagery. Importantly, disabled end-users are able to achieve similar performances as healthy participants.
\end{abstract}

Keywords: Brain-Computer Interfaces, electroencephalogram, motor imagery, shared control, robot, wheelchair

\section{Introduction}

Brain-Computer Interface (BCI) technology enables people to control machines not by manual operation, but by mere "thinking". Thereby, the electrical activity from the brain (the electroencephalogram, EEG) is analyzed in real-time and control parameters are extracted. BCIs are no longer only used by healthy subjects under standardized laboratory conditions, but are also introduced to end-users controlling applications in their homes [1]. Applications range from simple control of computers, by reestablishing communication via writing programs, to motor substitution and games. For people with motor disabilities, regaining mobility is a big issue. In this work, we want to present our latest results from two assistive mobility devices (a telepresence robot and an electric wheelchair) controlled by disabled end-users directly from a rehabilitation center.

\section{Methods}

Brain-Computer Interface and signal processing: All participants used motor imagery (e.g. of the left hand, right hand, feet) to control the BCI. Therefore, the brain activity was acquired via 16 EEG channels over the motor cortex. From the Laplacian filtered EEG, the power spectral density was calculated and classified with a Gaussian classifier (more details see [2]). The output of the BCI is visualized on a screen and used to control the application.

Application prototypes: In case of the telepresence application, the subject remotely controlled the robot, steering it to the left or to the right within an office environment [3]. If no commands were delivered, the default behavior of the robot was activated, which consisted of moving forward and avoiding obstacles with the help of a shared control system using its on-board sensors.

The second application was a powered wheelchair equipped with sonars and webcams, whose movements can be controlled similarly to the telepresence robot, except that the subject was co-located with it [4]. The subject had to drive the wheelchair to reach several targets.
Both applications were quite demanding for the subjects, since besides the increased workload and the split attention [2], a certain temporal precision was required.

\section{Results and Discussion}

Up to now, 9 end-users (aged $37.7 \pm 12.2$ year) tested the telepresence application. They achieved a ratio between the time needed to reach the targets with manual control vs. BCI control of $0.87 \pm 0.09$ (whereby 1 would correspond to perfect and 0 to no control). These performances are similar to healthy participants [3]. In case of the wheelchair only one person tested it and achieved an amazing 0.97.

Most importantly we could demonstrate that all end-users who achieved good BCI performance could also control the applications successfully with the help of the shared control system. This is very important, because having a good BCI control does not guarantee good control over the application, due to the necessary split attention between the application and the BCI, and the temporal constraints [2]. In the future we have to add a start/stop or a pausing functionality for the movement of the robotic device. In the framework of a hybrid BCI, such commands could also be delivered through other channels such as residual muscular activity, which can be controlled reliably-but not very often, because of the quick fatigue.

\section{Acknowledgement}

This work was supported by the European ICT Programme Project TOBI FP7-224631.

\section{Bibliography}

[1] J. d. R. Millán, R. Rupp, et. al., "Combining braincomputer interfaces and assistive technologies: Stateof-the-art and challenges," Front Neurosci, vol. 4, p. $161,2010$.

[2] R. Leeb, S. Perdikis, et.al., "Transferring braincomputer interface skills: from simple bci training to successful application control," Artif Intell Med, 2013, in revision.

[3] L. Tonin, T. Carlson, et. al., "Brain-controlled telepresence robot by motor-disabled people," in Conf Proc IEEE Eng Med Biol Soc, pp. 4227-4230, 2011.

[4] T. Carlson and J. d. R. Millán, "Brain-controlled wheelchairs: A robotic architecture," IEEE Robot Autom Mag, vol. 20, no. 1, pp. 65-73, 2013. 